\title{
Interstitial pneumonia induced by sorafenib in a patient with hepatocellular carcinoma: An autopsy case report
}

\author{
TAKASHI YAMAGUCHI ${ }^{1,2}$, TOSHIHITO SEKI ${ }^{1,2}$, CHIKA MIYASAKA $^{3}$, RYOSUKE INOKUCHI ${ }^{1,2}$, \\ RINAKO KAWAMURA $^{1,2}$, YUUTAKU SAKAGUCHI ${ }^{1,2}$, MIKI MURATA $^{1,2}$, KOICHI MATSUZAKI $^{1,2}$, \\ YORIKA NAKANO $^{3}$, YOSHIKO UEMURA ${ }^{3}$ and KAZUICHI OKAZAKI ${ }^{1}$ \\ ${ }^{1}$ Department of Gastroenterology and Hepatology, Kansai Medical University; ${ }^{2}$ Liver Disease Center, \\ Kansai Medical University Takii Hospital; ${ }^{3}$ Department of Diagnostic Pathology, \\ Kansai Medical University, Osaka 570-8507, Japan
}

Received March 13, 2014; Accepted November 13, 2014

DOI: 10.3892/ol.2015.2934

\begin{abstract}
Sorafenib is a multikinase inhibitor currently approved in Japan for the treatment of unresectable hepatocellular carcinoma. Interstitial pneumonia induced by sorafenib may have a fatal outcome, and therefore, has recently been the focus of many studies. The current report presents an autopsy case of diffuse alveolar damage (DAD) that occurred in a 59-year-old male, who had been treated with sorafenib. The patient had been given sorafenib for six months and had exhibited no respiratory symptoms during this time. However, 19 days after sorafenib treatment was resumed, acute interstitial pneumonia developed. In previously reported cases, the first symptoms of pulmonary toxicity appeared following a limited treatment duration with sorafenib; this was in contrast to the patient in the current study, who developed the first symptoms after eight months. We therefore conclude that physicians must be aware of interstitial pneumonia as a potential pulmonary toxicity associated with sorafenib treatment when treatment with sorafenib is resumed, even after prolonged use. In addition, to best of our knowledge, this is the first case of a postmortem examination reported in patient with interstitial pneumonia induced by sorafenib treatment.
\end{abstract}

\section{Introduction}

Sorafenib (Bayer Pharmaceuticals, West Haven, CT, USA) is a multikinase inhibitor, which functions by blocking tumor-cell proliferation and angiogenesis (1). In cases of advanced hepatocellular carcinoma (HCC) where patients received sorafenib

Correspondence to: Professor Toshihito Seki, Department of Gastroenterology and Hepatology, Kansai Medical University, 10-15 Fumizonocho, Moriguchi, Osaka 570-8507, Japan

E-mail: sekit@takii.kmu.ac.jp

Key words: diffuse alveolar damage, hepatocellular carcinoma, drug-induced interstitial pneumonia, molecular-targeted agent, sorafenib treatment, almost a 3-month median survival benefit was reported, as compared with patients receiving a placebo (2).

Common adverse side effects of sorafenib treatment include diarrhea, weight loss, skin rash (including hand-foot skin reactions), fatigue, and hypertension. Additionally, a number of cases of sorafenib-induced interstitial pneumonia have also been reported (3-5). Safety information for sorafenib therapy in patients with HCC was presented in Japan in October 2012, and six cases of acute respiratory failure were reported among 1,045 patients with HCC who had been treated with sorafenib (6). The current study describes an autopsy case of interstitial pneumonia that developed after the long-term treatment of a patient with advanced HCC with sorafenib. Written informed consent was obtained from the family of the patient.

\section{Case report}

A 59-year-old male with hepatitis C virus-related, multinodular HCC, exhibited progressive disease following eight sessions of transarterial chemoembolization (TACE) and four sessions of ablation therapy over the previous 15 years Kansai Medical University Takii Hospital (Osaka, Japan) and was admitted to the Department of Gastroenterology and Hepatology, Kansai Medical University (Osaka, Japan). Radiological studies showed growth of the tumor in the right lobe of the liver with several intrahepatic metastases, and further metastases to the lung. Although the patient had smoked until 25 years of age, no respiratory symptoms prior to the administration of sorafenib were observed. Additional medication at the time of commencing sorafenib treatment included ursodeoxycholic acid, branched-chain amino acid-containing pharmaceutical granular preparation, and tamsulosin hydrochloride. Fig. 1 shows the clinical course following the administration of sorafenib. Due to the patient's general state of health and Child-Pugh class B (score 7), palliative treatment with sorafenib (400 mg daily) was initiated in November 2011. After one week, the dosage was increased to $600 \mathrm{mg} /$ day. Two weeks following initiation, the administration of sorafenib was discontinued due to hand-foot-skin reaction, and was resumed at a dose of $400 \mathrm{mg} /$ day four weeks later. After five months, sorafenib treatment was discontinued again due to the patient being treated 
Table I. Laboratory data on admission.

\begin{tabular}{lc}
\hline Marker & Measurement \\
\hline Hematology & \\
WBC & $7,300 / \mu \mathrm{l}$ \\
Neutro & $90.5 \%$ \\
Lympho & $4.5 \%$ \\
Mono & $4.0 \%$ \\
Eosino & $0.5 \%$ \\
Baso & $0.5 \%$ \\
RBC & $350 \times 10^{4} / \mu 1$ \\
Hb & $8.6 \mathrm{~g} / \mathrm{dl}$ \\
Ht & $28.1 \%$ \\
Plt & $8.9 \times 10^{4} / \mu 1$ \\
Coagulation & \\
PT & $34 \%$ \\
INR & 1.79 \\
Biochemistry & \\
AST & $237 \mathrm{U} / 1$ \\
ALT & $89 \mathrm{U} / 1$ \\
T-Bil & $2.6 \mathrm{mg} / \mathrm{dl}$ \\
D-Bil & $1.5 \mathrm{mg} / \mathrm{dl}$ \\
ALP & $428 \mathrm{U} / 1$ \\
L-GTP & $17 \mathrm{U} / 1$ \\
TP & $1,119 \mathrm{U} / \mathrm{l}$ \\
Alb & $6.6 \mathrm{~g} / \mathrm{dl}$ \\
BUN & $2.1 \mathrm{~g} / \mathrm{dl}$ \\
Creatine & $25 \mathrm{mg} / \mathrm{dl}$ \\
CRP & $0.92 \mathrm{mg} / \mathrm{dl}$ \\
NH ${ }_{3}$ &
\end{tabular}

Tumor markers
AFP
AFP-L3
$6,139.0 \mathrm{ng} / \mathrm{ml}$
$65.1 \%$
PIVKA-II
$27,800 \mathrm{AU} / 1$

Blood gas analysis

$\begin{array}{lc}\mathrm{pH} & 7.394 \\ \mathrm{pCO}_{2} & 28.5 \mathrm{mgHg} \\ \mathrm{pO}_{2} & 62.5 \mathrm{mgHg} \\ \mathrm{HCO}_{3}{ }^{-} & 17.0 \mathrm{mEq} / \mathrm{l}\end{array}$

WBC, white blood cells; Hb, hemoglobin; Ht, hematocrit; Plt, platelet; PT, prothrombin time; INR, international normalized ratio; AST, aspartate transaminase; ALT, alanine transaminase; T-Bil, total bilirubin; D-Bil, direct bilirubin; ALP, alkaline phosphatase; $\gamma$-GTP, $\gamma$-glutamyl transferase; LDH, lactate dehydrogenase; TP, total protein; Alb, albumin; BUN, blood urea nitrogen; CRP, C-reactive protein; AFP, $\alpha$-fetoprotein; AFP-L3, AFP-L3 isoform; PIVKA-II, proteins induced by vitamin $\mathrm{K}$ absence or agonist-II; $\downarrow$, lower than normal range; $\uparrow$, higher than normal range.

with TACE, and subsequently resumed at $400 \mathrm{mg} / \mathrm{day}$ after four weeks. At 19 days following the treatment resumption,

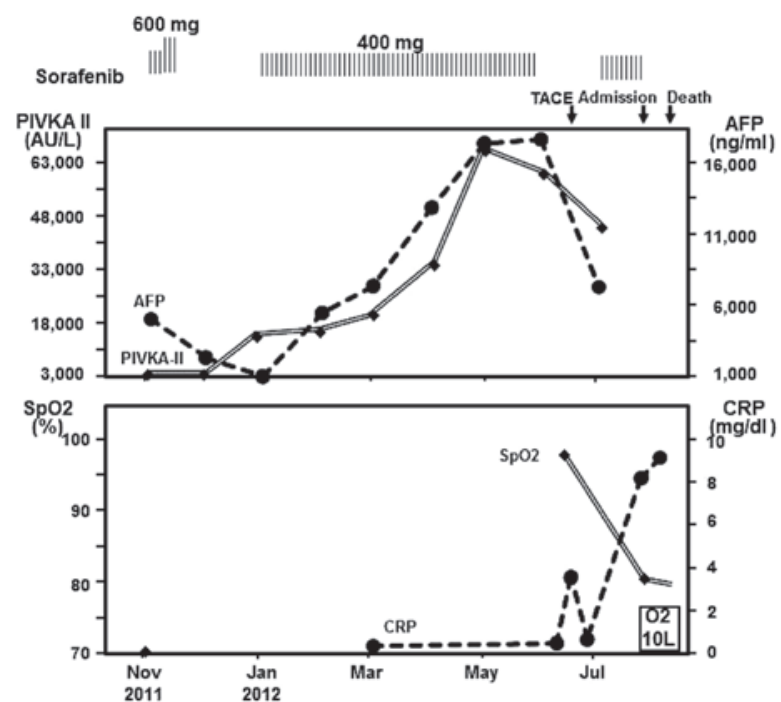

Figure 1. Clinical course of the patient after the administration of sorafenib. Serum AFP and PIVKA-II reduced after TACE. On day 19 of sorafenib readministration, the patient developed progressive dyspnea, and was admitted to hospital. The oxygen saturation was $81 \%$ despite oxygen supplementation. There was a clinical worsening, and the patient succumbed to the disease three days after admission. AFP, $\alpha$-fetoprotein; PIVKA-II, protein induced by vitamin $\mathrm{K}$ absence or antagonist II; TACE, transcatheter arterial chemoembolization; CRP, C-reactive protein.

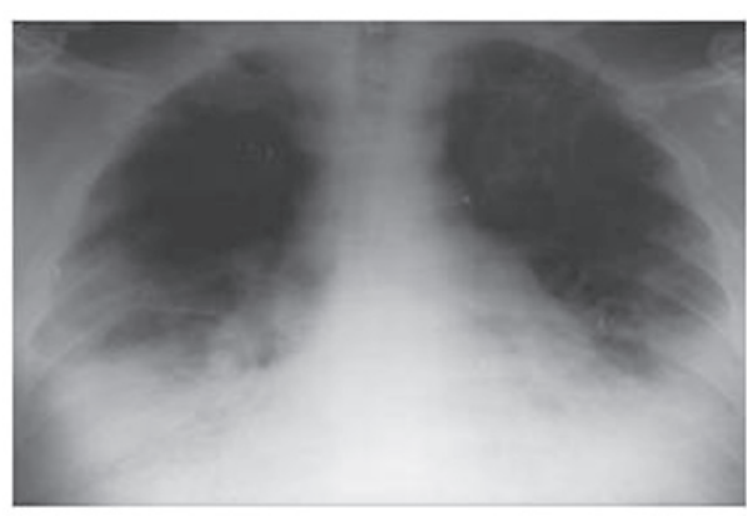

Figure 2. Chest X-ray on admission.

the patient developed progressive dyspnea and fever, with worsening general weakness, and presented to the emergency department of Kansai Medical University Takii Hospital with dyspnea, cough and fever. Analysis of the vital signs showed a normal blood pressure of $124 / 65 \mathrm{mmHg}$ (normal range, $100-129 / 60-80 \mathrm{mmHg}$ ), respiratory rate of 20 breaths $/ \mathrm{min}$ (normal range, $12-15$ breaths/min), pulse of 120 beats $/ \mathrm{min}$ (normal range, $60-85$ beats $/ \mathrm{min}$ ), and body temperature of $37.5^{\circ} \mathrm{C}$ (normal range, $35.0-37.0^{\circ} \mathrm{C}$ ). Respiratory crackles were audible in the bilateral lower lung fields; the patient was anemic and icteric. The air pulse oximetric saturation was $81 \%$ (normal limit, $>92 \%$ ); arterial blood gas analysis showed a $\mathrm{PaO}_{2}$ of $62.5 \mathrm{mmHg}$ (normal range, $80-100 \mathrm{mmHg}$ ); $\mathrm{PaCO}_{2}$ of $28.5 \mathrm{mmHg}$ (normal range, $35-45 \mathrm{mmHg}$ ) and $\mathrm{pH} 7.39$ (normal range, 7.35-7.45), despite oxygen supplementation. Laboratory studies showed marked leukocytosis with a white blood cell count of 7,300 cells $/ \mu 1$ (normal range, 3,5008,500 cells $/ \mu 1$ ), a neutrophil level of 6,607 cells $/ \mu 1$ (normal 


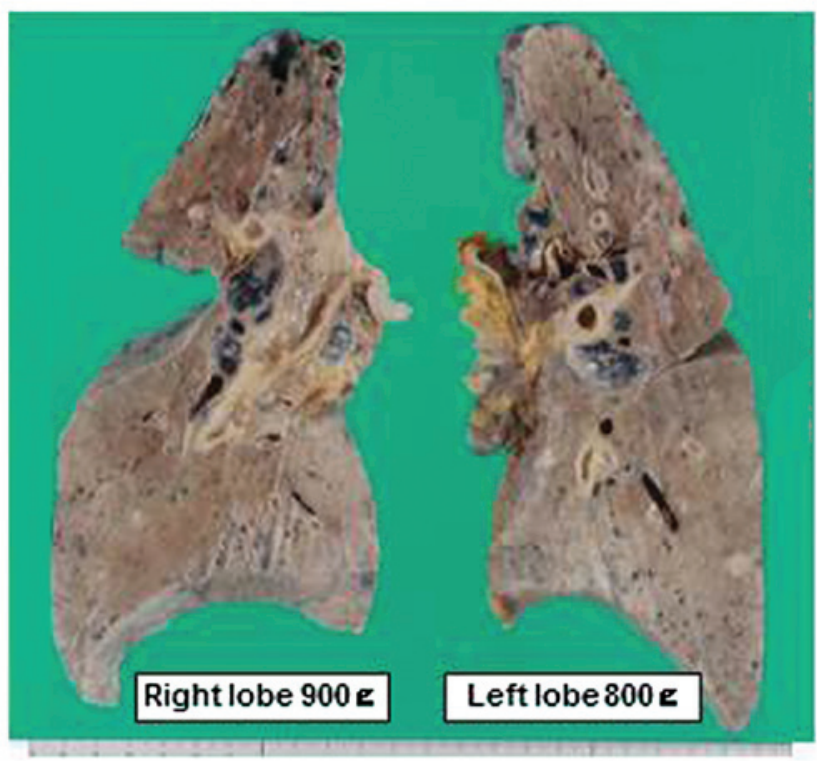

Figure 3. Gross features of the lungs at autopsy.

range, 1,470-6,545 cells/ $\mu \mathrm{l})$ and an elevated C-reactive protein level of $8.05 \mathrm{mg} / \mathrm{dl}$ (normal limit, $<0.3 \mathrm{mg} / \mathrm{dl}$ ); elevated aspartate transaminase concentration of 237 IU/1 (normal range, 13-35 IU/1), and alanine transaminase concentration of $89 \mathrm{IU} / 1$ (normal range, 5-35 IU/l) (Table I). Chest X-ray radiography revealed heart enlargement and bilateral pleural effusion, leading to a diagnosis of acute heart failure (Fig. 2). Sorafenib treatment was discontinued on admission to Kansai Medical University Takii Hospital as oral administration was difficult. The patient developed rapidly worsening dyspnea and hypoxia in spite of therapy with diuretic treatment and providing oxygen, and the patient succumbed to the disease three days following admission. The patient had declined mechanical ventilation. The autopsy was conducted with the consent of the family.

Autopsy findings. Gross findings of the autopsied liver revealed cirrhosis, and multiple nodular lesions, with the largest measuring $4 \mathrm{~cm}$ in diameter, were homogeneously yellow-white to green. Histologically, the lesions were composed of HCC and intrahepatic cholangiocarcinoma (ICC) elements. A histological diagnosis of intermixed HCC-ICC was determined. The HCC element revealed a proliferating trabecular pattern with bile production, corresponding to moderate differentiation as Edmondson's grade II (7). The ICC element was well-differentiated, forming a well-developed gland. The largest mass was widely necrotic and exhibited fibrotic changes, considered to be the effect of sorafenib treatment and TACE; the remaining masses were ICC elements. Metastasis to the lungs, hilar lymph nodes, and mediastinal lymph nodes was observed. Furthermore, necrosis was identified, partially due to sorafenib treatment, and the residual regions showed ICC elements only. On autopsy, the lungs were swollen, with a combined weight of $1,700 \mathrm{~g}$, and consolidated with a diffusely glistening spongy cut surface (Fig. 3). Histologically, the alveoli were obliterated by the hyaline membranes and organization of exudates with proliferation of fibroblasts, indicating diffuse alveolar damage (DAD). Notably, the bilateral lungs

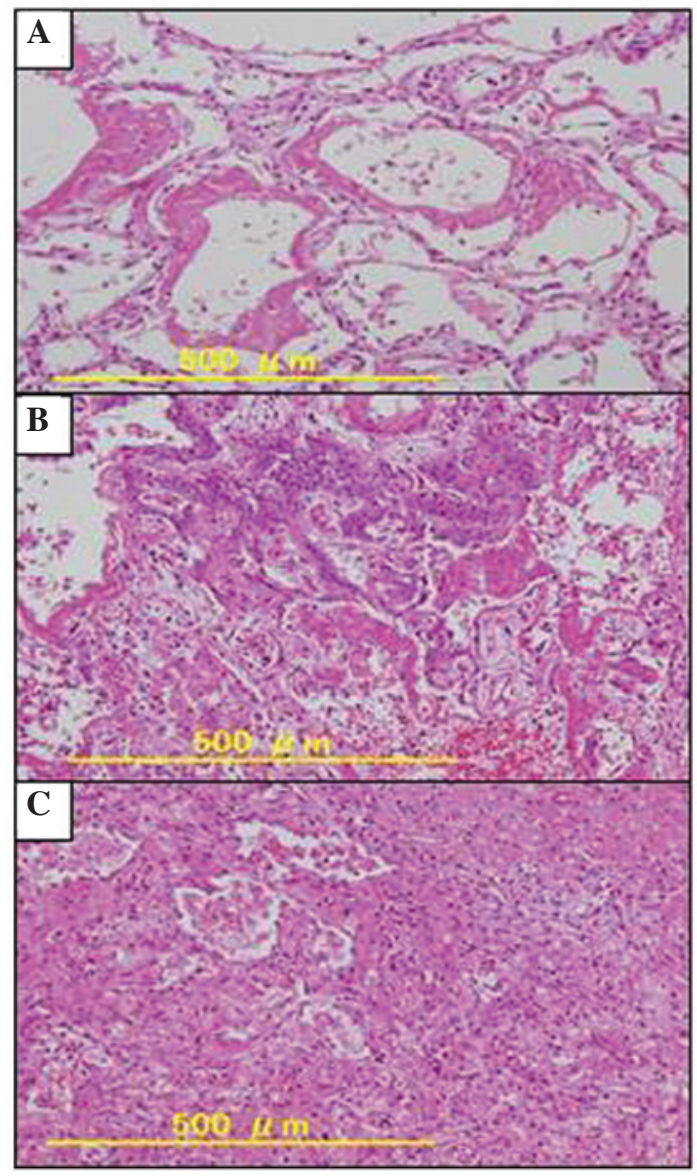

Figure 4. Microscopic findings in the lungs. Diffuse alveolar damage with hyaline membranes superimposed on a fibrotic lung background (hematoxylin and eosin stain). (A) Early exudative stage: Injury of type II pneumocytes with sloughing into alveolar lumens and hyaline membrane formation. (B) Proliferative stage: Organization of exudates composed of proliferating type II pneumocytes and fibroblasts with squamous metaplasia. (C) Late organizing fibrotic phase: Interstitial fibrosis with widening of alveolar septa and disappearance of the hyaline membranes.

showed a diffusely different phases of DAD, with the hyaline membrane producing an early exudative phase (Fig. 4A), a proliferative phase (Fig. 4B), and late organizing fibrotic phase (Fig. 4C). The mixed features of various phases were proposed to correspond to drug-induced DAD. Honeycombing was not observed. The findings did not indicate other organisms such as bacteria, cytomegalovirus, Pneumocystis jirovecii, and fungus. In addition, no vascular changes of pulmonary hypertension with a plexiform lesion were identified, however, a number of arterializations of small blood vessels were revealed. No evidence of recent myocardial infarction or acute cardiac decompensation was identified. Due to all of the results, a clinical diagnosis of fatal interstitial pneumonia associated with sorafenib treatment was determined.

\section{Discussion}

The current study presents an autopsy case involving a patient with advanced HCC who developed rapidly progressive interstitial lung disease following resumption of treatment with sorafenib. In the absence of other etiologies, and due to the autopsy findings, this patient was considered to have sorafenib-induced interstitial pneumonia. 
Histologically, the autopsied lungs revealed DAD, which is the morphological precursor to acute interstitial pneumonia and is characterized by a rapid and fatal clinical course. DAD manifests clinically as acute respiratory distress syndrome (ARDS) (8); it may be observed in sepsis, shock, trauma, severe ARDS, and idiopathic cases with undetected etiological factors as well as acute exacerbations of chronic interstitial lung diseases. While diffuse bilateral opacity is often observed on lung radiology, numerous cases display deep hypoxemia that requires mechanical ventilation; the mortality rate is $43-50 \%(9,10)$. A number of drugs have been associated with lung injury with a DAD pattern. The clinical features of lung toxicity are not specific (dyspnea, cough, fever, pulmonary infiltrates), and the differential diagnosis includes infection, relapse of the underlying disease, pulmonary edema, and changes due to oxygen or radiation (11). The pathological findings of drug-related DAD are also nonspecific and the diagnosis is one of exclusion (12). In the present case, the clinical history and exclusion of other causative factors indicate that sorafenib is the cause of lung injury.

Treatment with a number of molecular-targeted agents, including gefitinib, erlotinib, imatinib, and bortezomib, has been associated with pulmonary toxicity (13). However, the underlying mechanisms of how these molecular-targeted agents induce interstitial pneumonia remain unknown. The reduction of intrapulmonary vascular endothelial growth factor (VEGF) levels in the early stages of lung injury and normalization following recovery in ARDS have been confirmed in numerous studies $(14,15)$, as VEGF acts as a growth and anti-apoptotic factor on alveolar epithelial cells, in addition to its known effects on endothelial cells (16). Therefore, the pulmonary toxicity induced by sorafenib treatment may be associated with its mechanism of antitumor activity, involving the inhibition of the VEGF signaling pathway.

In previously reported cases, the initial symptoms of pulmonary toxicity appeared after a limited treatment duration with sorafenib (one to six weeks) (3-5). By contrast, the present case showed delayed onset after eight months of sorafenib treatment. The patient had been treated with sorafenib for six months with no respiratory symptoms prior to resuming sorafenib treatment. After 19 days of resuming the treatment, however, the patient developed acute interstitial pneumonia. A number of immune-related mechanisms in the interstitial pneumonia may be induced by sorafenib.

In conclusion, severe respiratory failure with a histological pattern of DAD may develop following resumption of treatment with sorafenib. Therefore, physicians must be aware of interstitial pneumonia as a potential pulmonary toxicity associated with sorafenib treatment, when a patient resumes treatment with sorafenib, even after prolonged use.

\section{References}

1. Wilhelm SM, Carter C, Tang L, et al: BAY 43-9006 exhibits broad spectrum oral antitumor activity and targets the $\mathrm{RAF} / \mathrm{MEK} / \mathrm{ERK}$ pathway and receptor tyrosine kinases involved in tumor progression and angiogenesis. Cancer Res 64: 7099-7109, 2004

2. Llovet JM, Burroughs A, and Bruix J: Hepatocellular carcinoma. Lancet 362: 1907-1917, 2003.

3. Ide $\mathrm{S}$, Soda $\mathrm{H}$, Hakariya $\mathrm{T}$, et al: Interstitial pneumonia probably associated with sorafenib treatment: An alert of an adverse event. Lung Cancer 67: 248-250, 2010.

4. Myung HJ, Jeong SH, Kim JW, et al: Sorafenib-induced interstitial pneumonitis in a patient with hepatocellular carcinoma: a case report. Gut Liver 4: 543-546, 2010.

5. Takeda H, Nishikawa H, Iguchi E, et al: Sorafenib-induced acute interstitial pneumonia in patients with advanced hepatocellular carcinoma: report of three cases. Clin J Gastroenterol 5: 407-412, 2012.

6. Bayer Healthcare Japan. Safety information for Nexabar ${ }^{\mathrm{TM}}$ 200mg tablets. June 2012 (In Japanese). http://www.nexavar. jp/ja/home/usage-information/servey-record/hcc/. Accessed March 10, 2014.

7. Edmondson HA and Steiner PE: Primary carcinoma of the liver: a study of 100 cases among 48,900 necropsies. Cancer 7: 462-503, 1954.

8. Myers JL and Katzenstein AL: Beyond a consensus classification for idiopathic interstitial pneumonias: progress and controversies. Histopathology 54: 90-103, 2009.

9. Esteban A, Fernández-Segoviano P, Frutos-Vivar F, et al: Comparison of clinical criteria for the acute respiratory distress syndrome with autopsy findings. Ann Intern Med 141: 440-445, 2004.

10. Zambon $\mathrm{M}$ and Vincent JL: Mortality rates for patients with acute lung injury/ARDS have decreased over time. Chest 133: 1120-1127, 2008.

11. Barber NA and Ganti AK: Pulmonary toxicities from targeted therapies: a review. Target Oncol 6: 235-243, 2011

12. Muller NL, White DA, Jiang H, et al: Diagnosis and management of drug-associated interstitial lung disease. Br J Cancer 91 Suppl 2: S24-S30, 2004.

13. Schwaiblmair M, Behr W, Haeckel T, et al: Drug induced interstitial lung disease. Open Respir Med J 6: 63-74, 2012.

14. Abadie Y, Bregeon F, Papazian L, et al: Decreased VEGF concentration in lung tissue and vascular injury during ARDS. Eur Respir J 25: 139-146, 2005.

15. Vasakova M, Sterclova M, Kolesar L, et al: Bronchoalveolar lavage fluid cellular characteristics, functional parameters and cytokine and chemokine levels in interstitial lung diseases. Scand J Immunol 69: 268-274, 2009.

16. Roberts JR, Perkins GD, Fujisawa T, et al: Vascular endothelial growth factor promotes physical wound repair and is antiapoptotic in primary distal lung epithelial and A549 cells. Crit Care Med 35: 2164-2170, 2007. 